\title{
Effects of RNAi-induced Skp2 inhibition on cell cycle, apoptosis and proliferation of endometrial carcinoma cells
}

\author{
HAO LIN ${ }^{1 *}$, GUAN-YU RUAN ${ }^{2 *}$, XIAO-QI SUN ${ }^{1}$, XIAO-YING CHEN ${ }^{1}$, XIU ZHENG $^{3}$ and PENG-MING SUN ${ }^{1,2}$ \\ ${ }^{1}$ Department of Gynecology, Fujian Provincial Maternity and Children's Hospital; ${ }^{2}$ Laboratory of Gynecologic Oncology, \\ Fujian Provincial Maternity and Children's Hospital, Affiliated Hospital of Fujian Medical University; \\ ${ }^{3}$ Department of Obstetrics and Gynecology, The First Affiliated Hospital of Fujian Medical University, \\ Fuzhou, Fujian 350001, P.R. China
}

Received June 12, 2018; Accepted February 11, 2019

DOI: $10.3892 / \mathrm{etm} .2019 .7392$

\begin{abstract}
The aim of the current study was to investigate the underlying mechanism of S-phase kinase associated protein 2 (Skp2) gene inhibition by lentivirus-mediated RNA interference (RNAi) on the cell cycle, apoptosis and proliferation of endometrial carcinoma HEC-1-A cells. A lentivirus shRNA vector targeting Skp2 was constructed and transfected into HEC-1-A cells. HEC-1-A cells transfected with a scramble sequence were used as negative controls. The mRNA and protein expression of Skp2, p27, cyclin D1 and caspase-3 were detected via reverse transcription-quantitative polymerase chain reaction and western blotting, respectively. The effects of Skp2 inhibition on the cell cycle, apoptosis and proliferation of HEC-1-A cells were detected using flow cytometry and a cell counting kit-8. Skp2 co-expression data was analyzed using Oncomine and TCGA databases. The positive recombinant viral clones were identified via PCR and confirmed via sequencing. The mRNA and protein expression of Skp2 were significantly decreased in HEC-1-A cells transfected with the lentiviral vectors compared with the negative control. In addition, there were no significant changes in the mRNA expression of $\mathrm{p} 27$ and cyclin D1; however, the protein levels of p27 and cyclin D1 were upregulated and downregulated, respectively, in HEC-1-A cells transfected with lentiviral vectors compared with negative controls. RNAi-induced Skp2 inhibition exerted an anti-proliferative effect by inducing cell cycle arrest, however cell apoptosis was not significantly affected. In the TCGA
\end{abstract}

Correspondence to: Professor Peng-Ming Sun, Laboratory of Gynecologic Oncology, Fujian Provincial Maternity and Children's Hospital, Affiliated Hospital of Fujian Medical University, 18 Daoshan Road, Fuzhou, Fujian 350001, P.R. China

E-mail: fmsun1975@fjmu.edu.cn

*Contributed equally

Key words: endometrial neoplasms, RNA interference, S-phase kinase associated protein 2, cell cycle, cell proliferation database, Skp2 expression positively associated with IGF2R, IGF2BP3, IGFBP1 and CCNF, while Skp2 expression negatively associated with IGF2, IGFBP6, IGFBP7 and IGFBP3. RNAi-induced Skp2 inhibition upregulated the protein expression of $\mathrm{p} 27$ and downregulated the protein expression of cyclin D1. The expression of Skp2 in endometrial cancer may therefore be regulated by the insulin-like growth factor 1 receptor signaling pathway.

\section{Introduction}

Endometrial cancer is one of the most common invasive malignancies of the female genital tract. The incidence of endometrial cancer has gradually increased, approaching or even surpassing that of cervical cancer, posing a serious threat to women's health (1). Surgery is the standard treatment for endometrial cancer, followed by chemotherapy, radiotherapy or hormone (progesterone) therapy (1). However, the prognosis of patients with advanced endometrial cancer remains poor following standard treatment and is often associated with significant side effects, including myelosuppression, liver damage, radiation enteritis and radiation cystitis (1). In recent years, gene therapy has become increasingly common in clinical cancer research (2). Gene therapy has several advantages, which include high selectivity and efficacy on metastases in advanced cancer, making it a promising therapeutic strategy for the treatment of patients with advanced cancer and a possible alternative to surgery, radiotherapy and chemotherapy (2).

S-phase kinase-associated protein 2 (Skp2) is a substrate recognition component of the $\mathrm{E} 3$ ubiquitin-protein ligase complex SCF (Skpl-Cullin-F-box) that recognizes specific phosphorylated substrates and mediates the ubiquitination and subsequent degradation of cellular regulatory factors involved in regulating cell cycle progression (3), signal transduction and transcription. Several cell cycle regulators are substrates of the ubiquitin proteasome pathway, which include cyclin D1 and p27 (4). Skp2 is involved in cell signal transduction and transcriptional regulation and is closely associated with several oncogenes and tumor suppressor genes, indicating that Skp2 possesses oncogenic potential (5). Skp2 serves a regulatory role in several processes including cell cycle regulation, 
growth, differentiation, proliferation, metastasis and apoptosis. Skp2 has also been reported to serve a role in drug resistance and is closely associated with the development of tumors, demonstrating a prognostic value in cancer (6-8). Skp2 is overexpressed in several types of human cancer, and it therefore targeting Skp2 may be a promising strategy for cancer treatment (9-11). Several studies have demonstrated that Skp2 expression is significantly increased in endometrial cancer compared with normal endometrium tissues (9-11). Skp2 is first produced in the G1/S phase of the cell cycle, increases in the $\mathrm{S} / \mathrm{G} 2$ phase and decreases rapidly in the M phase $(12,13)$. Skp2 thus exhibits time-dependent expression in the cell $(12,13)$. Therefore, targeted therapy may increase the sensitivity of chemotherapy and radiotherapy at specific cell cycle phases.

In the current study, Skp2 was selected as an RNAi target gene. Specific RNAi lentiviral expression vectors were constructed to inhibit Skp2 expression at the mRNA level to determine the effect of Skp2 inhibition on the characteristics of the endometrial cancer cell line, HEC-1-A, including the cell cycle, apoptosis and proliferation. The aim of the current study was to investigate the underlying mechanism of Skp2 in endometrial cancer progression as a potential for targeted gene therapy.

\section{Materials and methods}

Materials. HEC-1-A and 293T cell lines were obtained from Cell Bank of the Type Culture Collection of Chinese Academy of Sciences (Shanghai, China). The lentiviral vector system [which includes the lentiviral vector $\mathrm{pLV}$-green fluorescent protein (GFP), the lentivirus plasmid and two auxiliary packaging plasmids] was obtained from Shanghai GeneChem Co., Ltd. (Shanghai, China). T4 DNA ligase and restriction endonucleases were purchased from New England BioLabs, Inc., Ipswitch, MA, USA. A QIAGEN ${ }^{\circledR}$ Plasmid Extraction kit was purchased from Qiagen GmbH (Hilden, Germany). The PrimeScript ${ }^{\mathrm{TM}}$ RT reagent kit and SYBR ${ }^{\circledR}$ Premix Ex $\mathrm{Taq}^{\mathrm{TM}}$ were purchased from Takara Biotechnology Co., Ltd. (Dalian, China). PCR primer synthesis was performed by Shanghai Shenggong Biology Engineering Technology Service, Ltd. (Shanghai, China). Mouse anti-human Skp2 monoclonal antibodies were purchased from Invitrogen; Thermo Fisher Scientific, Inc. (Waltham, MA, USA). Mouse anti-human p27 monoclonal antibodies were purchased from Abcam (Cambridge, UK). Mouse anti-human cyclin D1 antibodies was purchased from Cell Signaling Technology, Inc. (Danvers, MA, USA). Mouse anti-human caspase-3 antibodies were purchased from Santa Cruz Biotechnology, Inc. (Dallas, TX, USA). A bicinchoninic acid (BCA) protein assay kit, radioimmunoprecipitation (RIPA) assay buffer, tetramethylethylenediamine, phenylmethylsulfonyl fluoride, a BeyoECL Plus kit, mouse anti-human $\beta$-actin antibodies, and horseradish peroxidase (HRP)-labeled goat anti-mouse IgG secondary antibodies were all purchased from Beyotime Institute of Biotechnology (Haimen, China). The Cell Cycle Assay kit [which includes propidium iodide and RNaseA] was purchased from Nanjing KeyGen Biotech Co., Ltd (Nanjing, China; cat. no. KGA512). The Cell Counting Kit-8 (CCK-8) assay was purchased from Tongren Chemical Institute (Kyushu, Japan).
Recombinant lentiviral vector construction. Two Skp2 transcript variants (ACCESSION NM_032637 and NM_005983) were identified in GenBank (https://www.ncbi.nlm.nih. gov/gene/6502) and the target was designed according to the homologous region. According to the design principle of RNA interference sequences, a pair of negative control nonsense sequences and four pairs of shRNA sequences were designed using BLOCK-iT ${ }^{\mathrm{TM}}$ RNAi Designer software from Ambion (Thermo Fisher Scientific, Inc.; https://rnaidesigner. thermofisher.com/rnaiexpress/). The constructs were named accordingly: Skp2-NC, Skp2-1, Skp2-2, Skp2-3 and Skp2-4. The siRNA target sequence was: Skp2-NC, 5'-TTCTCCGAACGT GTCACGT-3'; Skp2-1, 5'-CCTTAGACCTCACAGGTAA-3'; Skp2-2, 5'-CCAACCATTGGCTGAACAT-3'; Skp2-3, 5'-CAG AAAGAATCTCCAGAAA-3' and Skp2-4, 5'-GTGTCATGC TAAAGAATGA-3'. BLASTN was used to align the sequences with the corresponding human genome database, and homologous sequences of other coding sequences were excluded. The double-strand DNA oligonucleotide containing the interference sequence was synthesized by Shanghai GeneChem Co., Ltd. and cloned into the AgeI and EcoRI digested lentiviral vector pLV-GFP (part of the aforementioned lentiviral vector system). Upon transformation into E. coli competent cells, the positive clones were identified by PCR using the following upstream and downstream primers: 5'-CCTATTTCCCATGATTCCTTC ATA-3' and 5'-GTAATACGGTTATCCACGCG-3'. A stock solution containing $10 x$ buffer, $0.5 \mathrm{mM} \mathrm{MgCl}_{2}, 2.5 \mathrm{mM}$ dNTPs (Shanghai GeneChem Co., Ltd., Shanghai, China), $0.2 \mathrm{U} / \mu 1$, Taq DNA polymerase (Takara Biotechnology Co., Ltd., Dalian, China) and $0.4 \mu \mathrm{M}$ primers (Shanghai GeneChem Co., Ltd., Shanghai, China). A total of $20 \mu \mathrm{l}$ of PCR stock solution was added to each tube and the following thermocycling conditions were applied: $94^{\circ} \mathrm{C}$ for $2 \mathrm{~min}$, followed by 30 cycles of denaturation at $94^{\circ} \mathrm{C}$ for $30 \mathrm{sec}$, annealing at $60^{\circ} \mathrm{C}$ for $30 \mathrm{sec}$, elongation at $72^{\circ} \mathrm{C}$ for $30 \mathrm{sec}$ and final extension at $72^{\circ} \mathrm{C}$ for $7 \mathrm{~min}$. Agarose gel electrophoresis was then used to analyze the result. PCR primers were designed to reside within 2 separate DNA fragments so that a positive PCR band of expected size reflected the correct joining of 2 DNA fragments. For effective colony PCR and subsequent analysis by agarose gel, PCR product sizes of $\sim 343 \mathrm{bp}$ were found to be optimal. The positive clones were selected and sent to Shanghai Meiji Biotechnology Co., Ltd., (Shanghai, China) for sequencing analysis.

Lentivirus packaging. The recombinant lentivirus plasmid and two auxiliary packaging plasmids (provided by the aforementioned lentiviral vector system) were purified using the QIAGEN Plasmid Extraction kit, according to the manufacturer's protocol. Recombinant lentiviral particles were generated using 293T cells which were co-transfected using Lipofectamine ${ }^{\circledR} 2000$ (Invitrogen; Thermo Fisher Scientific, Inc.), according to the manufacturer's protocol. Following $48 \mathrm{~h}$ transfection, 293T cell supernatants rich in lentivirus particles were collected. After the supernatant was obtained, virus titers were determined in $293 \mathrm{~T}$ cells using an stepwise dilution method (14).

Cell culture and transfection of HEC-1-A cells. HEC-1-A cells were cultured in high glucose Dulbecco's modified Eagle's medium (DMEM; Gibco; Thermo Fisher Scientific, Inc.) supplemented with $100 \mathrm{U} / \mathrm{ml}$ penicillin-streptomycin and $10 \%$ 
newborn bovine serum (Zhejiang Tianhang Biotechnology Co., Ltd., Zhejiang, China), and cells were maintained at $37^{\circ} \mathrm{C}$ in a $5 \% \mathrm{CO}_{2}$-humidified incubator. The following groups were included: The negative control group (LV-Skp2-NC) and the experimental groups (LV-Skp2-1, LV-Skp2-2, LV-Skp2-3 and LV-Skp2-4). Cells were seeded into six-well plates at a density of $1 \times 10^{5}$ cells/well. Once cells reached $30 \%$ confluence, the recombinant lentiviruses LV-Skp2-1, LV-SKP-2, LV-Skp2-3 or LV-SKP-4 were used to transfect HEC-1-A cells ( $5 \cdot \mathrm{g} / \mathrm{ml}$ polybrene; Sigma-Aldrich; Merck KGaA, Darmstadt, Germany); multiplicity of infection, 10). Transfection efficiency was determined by observing GFP expression in HEC-1-A cells using an inverted fluorescence microscope (magnification, $\mathrm{x} 40$ ) under six fields of view.

Reverse transcription-quantitative polymerase chain reaction $(R T-q P C R)$. Total RNA was extracted from HEC-1-A cells on day 4 or 5 following recombinant lentiviral transfection using the TRIzol ${ }^{\circledR}$ reagent (Invitrogen; Thermo Fisher Scientific, Inc.). Total RNA $(2.5 \cdot \mathrm{g})$ was reversed transcribed into cDNA using the PrimeScript ${ }^{\mathrm{TM}} \mathrm{RT}$ reagent kit (Takara Biotechnology Co., Ltd.), according to the manufacturer's protocol. Subsequently, qPCR was performed using the SYBR $^{\circledR}$ Premix Ex Taq ${ }^{\mathrm{TM}}$ (Takara Biotechnology Co., Ltd.), according to the manufacturer's protocol. PRIMER3 5.0 software (PREMIER Biosoft International, Inc., Palo Alto, CA, USA) was used to design specific primers for Skp2, p27, Cyclin D1, and $\beta$-actin. The following primer pairs were used for qPCR: Skp2 forward, 5'-CCAGGAGATTCCAGACCT GAGT-3' and reverse, 5'-TGTCACTCCCTTTGCTCTTCA G-3' (212 bp); p27 forward, 5'-GGGGTATGAAGAGCTTGC TTTG-3' and reverse, 5'-GGGCAGTGAGGATAGGTTTCT G-3' (308 bp); cyclin D1 forward, 5'-TCAAATGTGTGCAGA AGGAGGT-3' and reverse, 5'-ATGGAGTTGTCGGTGTAG ATGC-3' (262 bp); $\beta$-actin forward, 5'-TCGTGCGTGACA TTAAGGAG-3' and reverse, 5'-AAGGTAGTTTCGTGGATG CC-3' (214 bp). RT-qPCR uses a two-step reaction according to the protocol of SYBR ${ }^{\circledR}$ PremixEx Taq ${ }^{\mathrm{TM}}$. The following thermocycling conditions were utilized: Initial denaturation at $95^{\circ} \mathrm{C}$ for $15 \mathrm{sec}$; 40 cycles of $95^{\circ} \mathrm{C}$ for $5 \mathrm{sec}$ and $60^{\circ} \mathrm{C}$ for $31 \mathrm{sec}$. The relative mRNA expression of Skp2, p27 and cyclin D1 were quantified using the $2^{-\Delta \Delta \mathrm{Cq}}$ method (15) and normalized to the internal reference gene, $\beta$-actin.

Western blot analysis. Total protein was extracted from HEC-1-A cells on day 7 following recombinant lentiviral transfection using RIPA lysis buffer and low-temperature centrifugation at $10,000 \mathrm{x}$ g for $10 \mathrm{~min}$ at $4^{\circ} \mathrm{C}$. Total protein was quantified using a BCA assay and $50 \cdot \mathrm{g}$ protein/lane was separated via SDS-PAGE on a $12 \%$ gel at $110 \mathrm{~V}$. The separated proteins were transferred onto polyvinylidene difluoride membranes and blocked with $10 \%$ skim milk solution at room temperature for 2-3 h. The membranes were then incubated with primary antibodies against Skp2 (1:500; cat. no. 32-3300), p27 (1:500; cat. no. ab54563), cyclin D1 (1:1,000; cat. no. 2926), caspase-3 (1:200; cat. no. sc-396225) or $\beta$-actin $\left(1: 1,000\right.$; cat. no. AA128) overnight at $4^{\circ} \mathrm{C}$. The membranes were washed with Tris-buffered saline containing $0.04 \%$ Tween-20 (TBST), followed by incubation with HRP-labeled secondary antibodies (1:2,000; cat. no. A0216) for $2 \mathrm{~h}$ at room temperature. The membranes were washed with TBST prior to chemiluminescence imaging. Protein bands were visualized using the BeyoECL Plus (Beyotime Institute of Biotechnology) and protein expression was quantified using the ImageScanner Imaging System (Amersham; GE Healthcare Life Sciences, Little Chalfont, UK) to process the film, Imagequant TL 7.0 image analysis software (Amersham; GE Healthcare Life Sciences, Little Chalfont, UK) was utilized to analyze the molecular weight and net optical density of the protein band.

Flow cytometry. Cell cycle and apoptosis assays were performed using a Beckman Coulter EPICS XL flow cytometer (Beckman Coulter, Inc., Brea, CA., USA). HEC-1-A cells were seeded into 6 -well plates at density of $1 \times 10^{5}$ cells/well and upon reaching $30 \%$ confluence, the negative control virus LV-Skp2-NC and recombinant lentiviruses LV-Skp2-3 and LV-Skp2-4 were transfected into HEC-1-A cells. A total of 6 days following infection, EDTA-free trypsin was added and the cells were harvested. Following centrifugation at $1,000 \mathrm{xg}$ for $5 \mathrm{~min}$, cell pellets were washed twice with pre-cooled PBS and fixed with $70 \%$ pre-cooled ethanol at $-20^{\circ} \mathrm{C}$ for $>12 \mathrm{~h}$. Following further centrifugation at $1,000 \mathrm{x} \mathrm{g}$ for $5 \mathrm{~min}$, ethanol was removed and cells were washed twice with PBS. Cells were resuspended with PBS, then propidium iodide and RNaseA were added at a final concentration of 20 and $50 \mu \mathrm{g} / \mathrm{ml}$, respectively, according to the instructions of the Cell Cycle Assay kit. Samples were then incubated for $30 \mathrm{~min}$ at $37^{\circ} \mathrm{C}$ in the dark. Analysis of the flow cytometric data were performed using System IITM 3.0 software (Beckman Coulter, Inc.). All experiments were performed in triplicate.

Cell proliferation assay. Cell proliferation was analyzed using a CCK-8 assay (Tongren Chemical Institute). HEC-1-A cells were seeded into 96-well plates at density of $5 \times 10^{3}$ cells/well in high glucose DMEM supplemented with $100 \mathrm{U} / \mathrm{ml}$ penicillin-streptomycin and $10 \%$ newborn bovine serum and once the cells reached $30 \%$ confluence, the negative control virus LV-Skp2-NC and recombinant lentiviruses LV-Skp2-3 and LV-Skp2-4 were transfected into HEC-1-A cells. The medium was replaced with DMEM supplemented with $10 \%$ newborn bovine serum ( $90 \cdot 1 /$ well) and $10 \cdot 1 \mathrm{CCK}-8$ reagent at 12,36 , $60,84,108,132$, and $156 \mathrm{~h}$ time points following infection and cells were incubated at $37^{\circ} \mathrm{C}$ in a $5 \% \mathrm{CO}_{2}$-humidified incubator for $30 \mathrm{~min}$. The absorbance was measured at a wavelength of $450 / 630 \mathrm{~nm}$ with a microplate reader (Bio-Rad Model 550; Bio-Rad Laboratories, Inc., Hercules, CA, USA). Three biological and three technical replicates were performed.

Bioinformatics analysis. Skp2 co-expression gene data was analyzed using Oncomine (www.oncomine.org) from published data obtained from obtained from Wu et al (16). The heatmap and the association between Skp2 and co-expressed genes in the cohort of patients with endometrial cancer in the TGCA database were analyzed using UCSC Xena (xena.ucsc.edu), and the expression data for each gene was used for further analysis.

Statistical analysis. Data presented as the mean \pm standard deviation. All statistical analyses were performed using SPSS statistical software (version 17.0; SPSS, Inc., Chicago, IL, 
USA). One-way analysis of variance followed by a Dunnett's post hoc test was used to analyze differences among multiple groups. A Student's t-test was used to analyze differences between the expression level of specific genes in the Skp2-high and Skp2-low endometrial cancer samples. $\mathrm{P}<0.05$ was considered to indicate a statistically significant difference.

\section{Results}

Transfection efficiency of recombinant lentivirus. Transfection efficiency was examined in HEC-1-A cells on day 7 following transfection with recombinant lentiviral vectors LV-Skp2-1, LV-Skp2-2, LV-Skp2-3, LV-Skp2-4 or LV-Skp2-NC. The percentage of cells expressing GFP (the transfection efficiency) observed under a fluorescent microscope was $>70 \%$ compared with cells observed under light microscopy (data not shown).

Skp2 expression following lentiviral transfection. The relative Skp2 mRNA expression level was determined via RT-qPCR in HEC-1-A cells on day 5 following transfection with recombinant lentiviral vectors (LV-Skp2-1, LV-Skp2-2, LV-Skp2-3, LV-Skp2-4 or LV-Skp2-NC). The mRNA expression of Skp2 was significantly decreased in cells transfected with LV-Skp2-3 or LV-Skp2-4 compared with the negative control, LV-Skp2-NC ( $<<0.01$; Fig. 1A). These results indicate that recombinant lentiviruses can specifically inhibit the expression of Skp2 mRNA, with inhibition rates of $54.39 \pm 3.19$, $69.87 \pm 2.88,80.36 \pm 2.61$ and $86.46 \pm 0.77 \%$, respectively (data not shown). The relative protein expression of Skp2 was determined by western blot analysis in HEC-1-A cells on day 7 following transfection with recombinant lentiviral vectors. The protein expression of Skp2 was significantly decreased in cells transfected with all four lentiviral vectors compared with cells transfected with the negative control $(\mathrm{P}<0.01$; Fig. 1B and $\mathrm{C})$. These results suggest that all four recombinant lentiviruses can influence Skp2 protein inhibition, with inhibition rates of $5.11 \pm 0.68,38.16 \pm 0.85,53.04 \pm 1.51$ and $55.34 \pm 0.97 \%$, respectively (data not shown). Furthermore, the inhibitory effect of LV-Skp2-3 and LV-Skp2-4 was greater than LV-Skp2-1 or LV-Skp2-2 (Fig. 1C). The two recombinant lentiviral vectors LV-Skp2-3 and LV-Skp2-4 could therefore inhibit the expression of Skp2 at the mRNA and protein level, achieving the greatest effect on Skp2 gene silencing.

Effect of Skp2 inhibition on cell cycle and apoptosis. Flow cytometry was used to detect the effect of RNA interference of (RNAi)-induced Skp2 inhibition on the cell cycle and apoptosis of HEC-1-A cells following transfection with recombinant lentiviral vectors LV-Skp2-3, LV-Skp2-4 or LV-Skp2-NC. The proportion of cells in the $G_{0} / G_{1}$ phase was significantly decreased, while the proportion of cells in the G2/M phase was significantly increased in cells transfected with LV-Skp2-3 or LV-Skp2-4 compared with LV-Skp2-NC $(\mathrm{P}<0.05$; Table I). In addition, there were no significant differences observed in the rate of apoptosis in cells transfected with LV-Skp2-3, LV-Skp2-4 or LV-Skp2-NC, 2.59 $\pm 0.10,2.68 \pm 0.15$ and $2.54 \pm 0.25 \%$, respectively (Fig. 2A). Furthermore, there were no significant differences in the protein expression of caspase-3 in cells transfected with LV-Skp2-3, LV-Skp2-4 or LV-Skp2-NC (Fig. 2B and C).
Table I. Effect of recombinant lentivirus interference on the cell cycle, as detected via flow cytometry.

\begin{tabular}{lccc}
\hline Group & $\mathrm{G}_{0} / \mathrm{G}_{1}$ & $\mathrm{~S}$ & $\mathrm{G}_{2} / \mathrm{M}$ \\
\hline LV-Skp2-NC & $40.00 \pm 4.90$ & $25.77 \pm 2.55$ & $34.23 \pm 2.35$ \\
LV-Skp2-3 & $32.63 \pm 0.83^{\mathrm{a}}$ & $25.23 \pm 0.65$ & $42.13 \pm 0.25^{\mathrm{a}}$ \\
LV-Skp2-4 & $30.26 \pm 1.55^{\mathrm{a}}$ & $26.40 \pm 0.80$ & $43.33 \pm 0.75^{\mathrm{a}}$ \\
\hline
\end{tabular}

Data presented as the mean \pm standard deviation. ${ }^{\mathrm{a}} \mathrm{P}<0.05$ vs. LVSkp2-NC. LV, lentivirus; Skp2, S-phase kinase associated protein 2.

Effect of RNAi-induced Skp2 inhibition on cell proliferation. Cell proliferation was assessed in HEC-1-A cells following transfection with the recombinant lentiviral vectors LV-Skp2-3 and LV-Skp2-4. Although there were no significant differences at 12,36 and $60 \mathrm{~h}$, cell proliferation at 132 and $156 \mathrm{~h}$ was significantly decreased in cells transfected with LV-Skp2-3 or LV-Skp2-4 compared with LV-Skp2-NC (P<0.01; Fig. 3A). In addition, cell proliferation significantly decreased at 84 and $108 \mathrm{~h}$ in cells transfected with LV-Skp2-4 compared with LV-Skp2-NC ( $\mathrm{P}<0.05$; Fig. 3A). The relative mRNA expression of cyclin D1 was determined via RT-qPCR in HEC-1-A cells on day 4 and 5 following transfection with recombinant lentiviral vectors LV-Skp2-3, LV-Skp2-4 or LV-Skp2-NC. The results revealed that there was no significant change in the mRNA expression of cyclin D1 (Fig. 3B). The relative protein expression of cyclin D1 was detected via western blotting in HEC-1-A cells on day 7 following transfection with recombinant lentiviral vectors (Fig. 3C). The protein expression of cyclin D1 was significantly decreased in cells transfected with LV-Skp2-4 compared with LV-Skp2-NC (P<0.01; Fig. 3D).

Changes in p27 expression following letiviral transfection. The relative mRNA expression of p27 was determined via RT-qPCR in HEC-1-A cells on day 4 and 5 following transfection with recombinant lentiviral vectors LV-Skp2-3, LV-Skp2-4 or LV-Skp2-NC. The results revealed that there was no significant change in the mRNA expression of p27 (Fig. 4A). The relative protein expression of $\mathrm{p} 27$ was detected via western blotting in HEC-1-A cells on day 7 following transfection with recombinant lentiviral vectors (Fig. 4B). The protein expression of p27 was significantly increased in cells transfected with LV-Skp2-3 or LV-Skp2-4 compared with LV-Skp2-NC transfected cells $(\mathrm{P}<0.01$; Fig. 4C).

Skp2 co-expressed genes. To further determine the underlying regulatory mechanism of Skp2 in endometrial cancer, data mining was performed using the Oncomine and TCGA databases. The co-expression data obtained from Wu et al (16) was analyzed using Oncomine. The results demonstrated that the genes involved in the insulin-like growth factor 1 receptor (IGFR) signaling pathway were enriched in the Skp2 co-expression gene dataset (data not shown). Heatmaps of these genes were generated and analyzed using UCSC Xena browser (Fig. 5A). The results demonstrated that the expression of Skp2 was associated with insulin-like growth factor 2 receptor (IGF2R), insulin-like growth factor 2 mRNA binding 
A

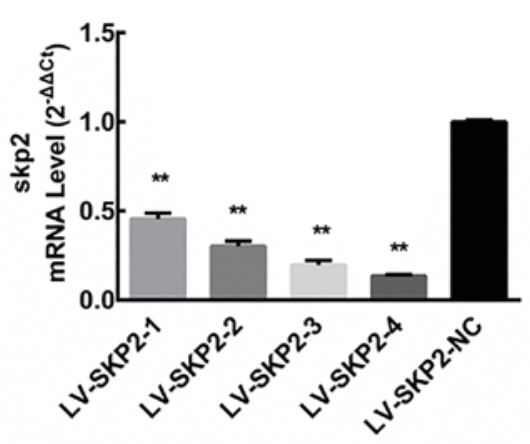

C

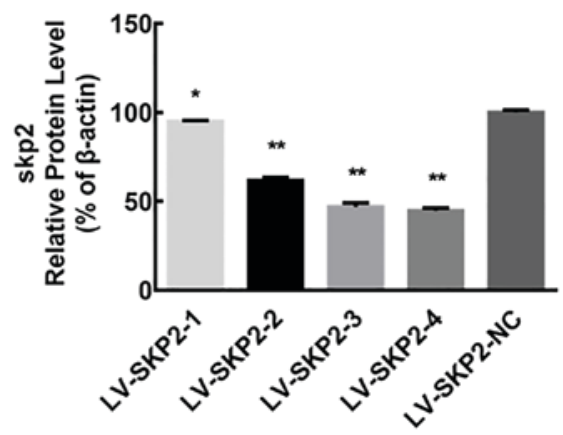

B

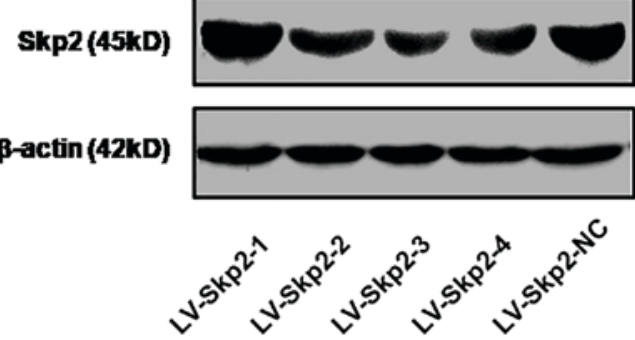

Figure 1. Skp2 expression was significantly decreased following lentiviral transfection. (A) Relative Skp2 mRNA levels was determined via reverse transcription-quantitative polymerase chain reaction in HEC-1-A cells on day 5 following transfection with recombinant lentiviral vectors LV-Skp2-1, LV-Skp2-2, LV-Skp2-3, LV-Skp2-4 or LV-Skp2-NC. (B) Relative protein levels of Skp2 was determined via western blot analysis in HEC-1-A cells on day 7 following transfection with recombinant lentiviral vectors. (C) Quantification of Skp2 protein expression. ${ }^{*} \mathrm{P}<0.05 ;{ }^{* *} \mathrm{P}<0.01$ vs. LV-Skp2-NC. Skp2, S-phase kinase associated protein 2; mRNA, microRNA; LV, lentivirus.

A

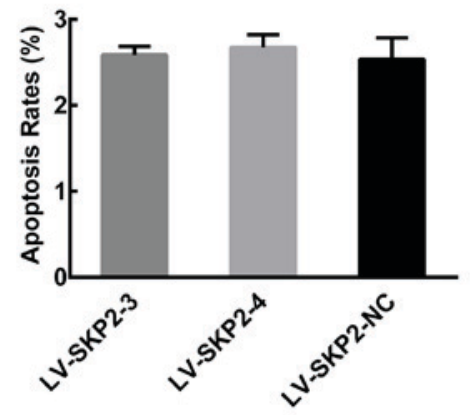

C

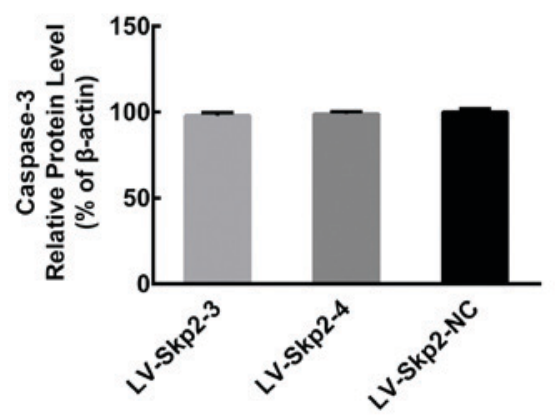

B

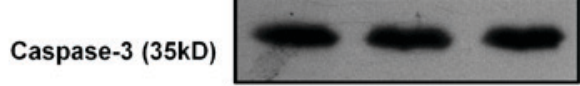

$\beta$-actin (42kD)
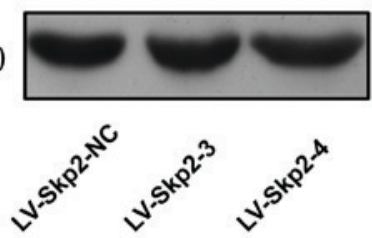

Figure 2. Effect of RNAi-induced Skp2 inhibition on cell apoptosis. (A) Flow cytometry was used to detect the effects of RNAi-induced Skp2 inhibition on cell apoptosis in HEC-1-A cells following transfection with recombinant lentiviral vectors LV-Skp2-3, LV-Skp2-4 or LV-Skp2-NC. (B) The relative protein expression of caspase-3 was determined via western blot analysis in HEC-1-A cells on day 7 following transfection with recombinant lentiviral vectors. (C) Quantification of Caspase-3 protein expression. RNAi, RNA interference; Skp2, S-phase kinase associated protein 2; LV, lentivirus.

protein 3 (IGF2BP3), insulin-like growth factor 2 mRNA binding protein 1 (IGFBP1) and cyclin $\mathrm{F}$ (CCNF), while no association was observed with insulin-like growth factor 2 (IGF2), insulin-like growth factor binding protein 6 (IGFBP6), 
A

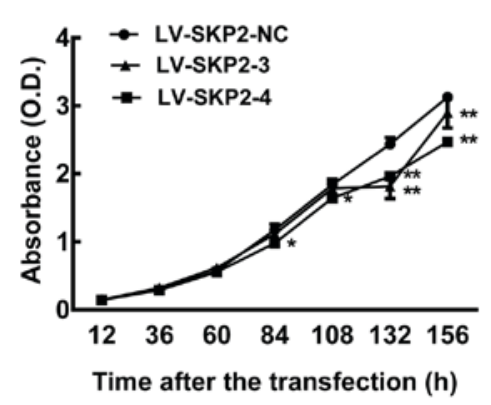

C

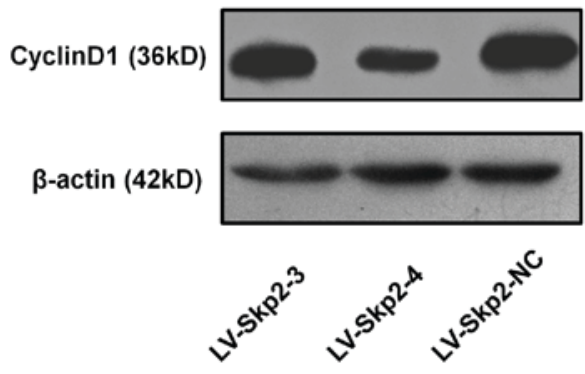

B

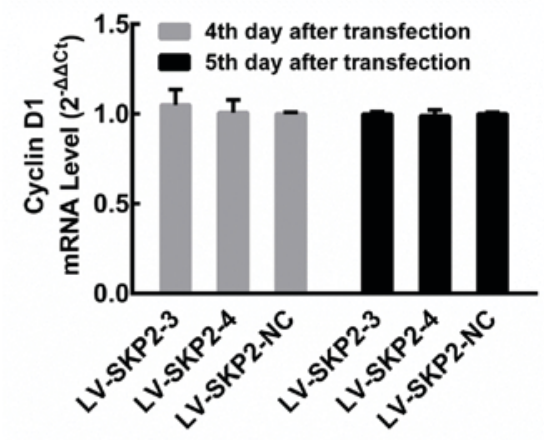

D

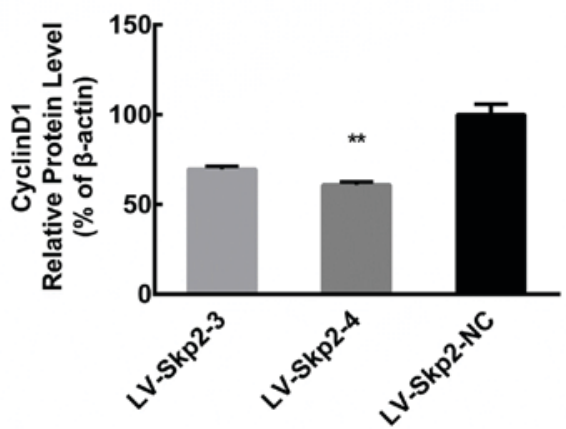

Figure 3. Effect of RNAi-induced Skp2 inhibition on cell proliferation. (A) Cell proliferation was examined in HEC-1-A cells following transfection with recombinant lentiviral vectors LV-Skp2-3, LV-Skp2-4 or LV-Skp2-NC. (B) Relative cyclin D1 mRNA levels were determined via reverse transcription-quantitative polymerase chain reaction in HEC-1-A cells on day 4 and 5 following transfection with recombinant lentiviral vectors LV-Skp2-3, LV-Skp2-4 or LV-Skp2-NC. (C) The relative protein expression of cyclin D1 was determined via western blot analysis in HEC-1-A cells on day 7 following transfection with recombinant lentiviral vectors. (D) Quantification of Cyclin D1 protein expression. ${ }^{*} \mathrm{P}<0.05 ;{ }^{* *} \mathrm{P}<0.01$ vs. LV-Skp2-NC. RNAi, RNA interference; Skp2, S-phase kinase associated protein 2; mRNA, microRNA; LV, lentivirus.

A
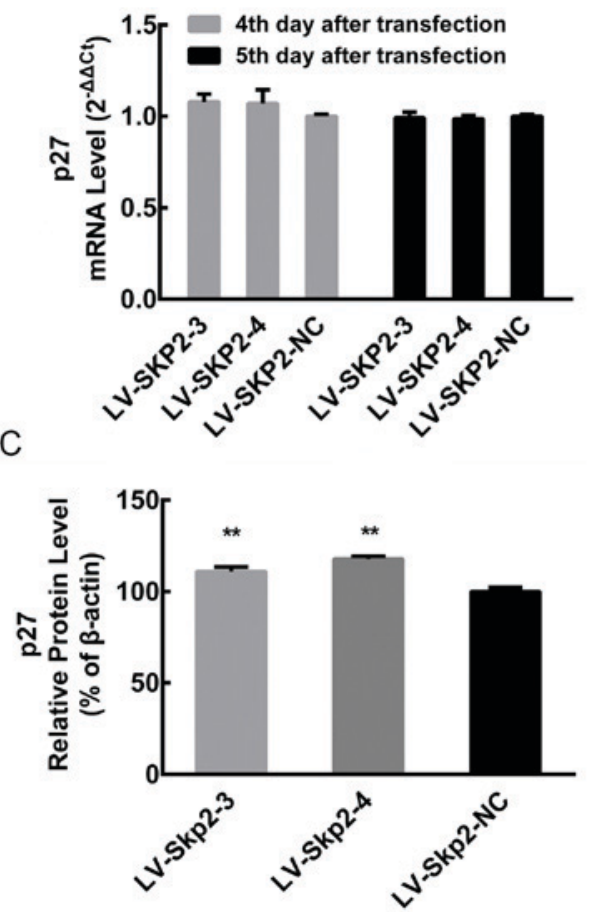

B

p27 (27kD)

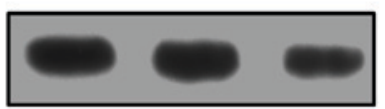

$\beta$-actin (42kD)
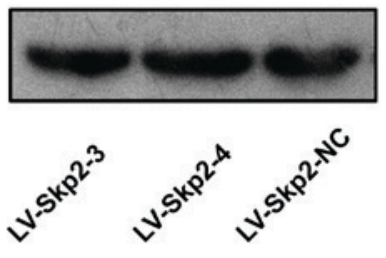

Figure 4. Effect of p27 expression following lentiviral transfection. (A) Relative p27 mRNA levels were determined via reverse transcription-quantitative polymerase chain reaction in HEC-1-A cells on day 4 and 5 following transfection with recombinant lentiviral vectors LV-Skp2-3, LV-Skp2-4 or LV-Skp2-NC. (B) The relative protein expression of p27 was determined via western blot analysis in HEC-1-A cells on day 7 following transfection with recombinant lentiviral vectors. (C) Quantification of 27 protein expression. ${ }^{* *} \mathrm{P}<0.01$ vs. LV-Skp2-NC. mRNA, microRNA; Skp2, S-phase kinase associated protein 2; LV, lentivirus. 
A

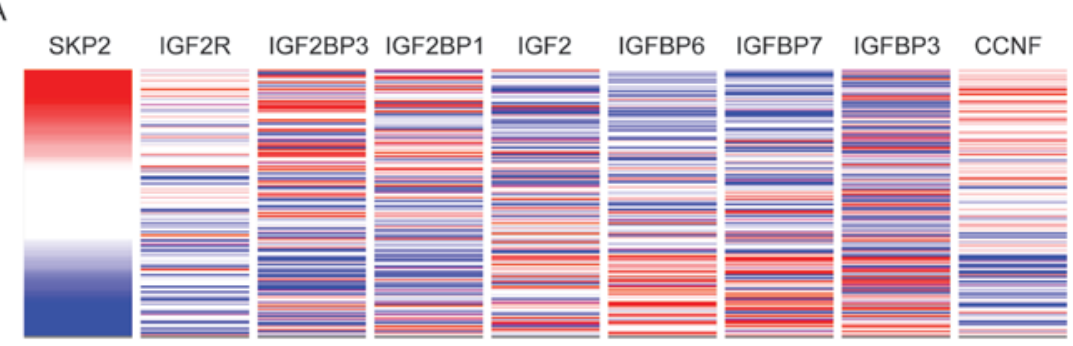

Low
Log2(Norm_Count+1)

B
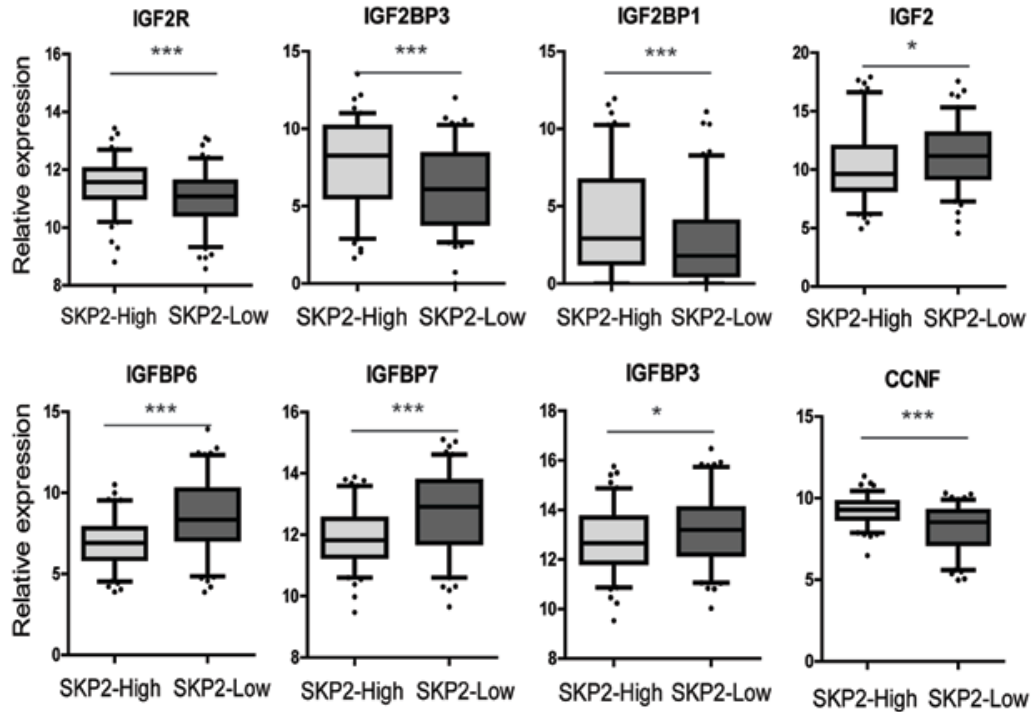

Figure 5. Skp2 co-expressed with genes in the IGFR signaling pathway. (A) Heatmap identification of gene co-expression patterns for Skp2, IGF2R, IGF2BP3, IGF2BP1, CCNF, IGF2, IGFBP6, IGFBP7 and IGFBP3 in the TCGA-EC dataset. (B) The relative gene expression of these genes in Skp2-High and Skp2-Low endometrial cancer samples. ${ }^{*} \mathrm{P}<0.05 ;{ }^{* * *} \mathrm{P}<0.001$ as indicated. Skp2, S-phase kinase associated protein 2; IGFR, insulin-like growth factor 1 receptor; IGF2R, insulin-like growth factor 2 receptor; IGF2BP3, insulin-like growth factor 2 mRNA binding protein 3; IGF2BP1, insulin-like growth factor 2 mRNA binding protein 1; CCNF, Cyclin F; IGF2, insulin-like growth factor 2; IGFBP6, insulin-like growth factor binding protein 6; IGFBP7, insulin-like growth factor binding protein 7; IGFBP3, insulin-like growth factor binding protein 3 .

insulin-like growth factor binding protein 7 (IGFBP7) and insulin-like growth factor binding protein 3 (IGFBP3; data not shown). In addition, there was no association observed between Skp2 and insulin like growth factor 1, IGFR and insulin-like growth factor $2 \mathrm{mRNA}$ binding protein 2 (data not shown). The gene expression data for the cohort of patients $(n=201)$ with endometrial cancer in the TGCA database were analyzed. The 201 TCGA-EC samples were divided into two groups: Skp2-High $(n=100)$ and Skp2-low $(n=101)$, according to the expression of Skp2 and the expression of each co-expressed gene. In the Skp2-High group, the expression of IGF2R $(\mathrm{P}<0.001)$, IGF2BP3 ( $<<0.001)$, IGF2BP1 $(\mathrm{P}<0.001)$ and $C C N F(P<0.001)$ were significantly increased, while the expression of IGF2 $(\mathrm{P}<0.05)$, IGFBP6 $(\mathrm{P}<0.001)$, IGFBP7 $(\mathrm{P}<0.001)$ and IGFBP3 $(\mathrm{P}<0.05)$ were significantly decreased compared with the Skp2-low group (Fig. 5B). Taken together, these results suggest that Skp2 may be regulated by the IGFR signaling pathway.

\section{Discussion}

The ubiquitin-proteasome system is an important mechanism for the regulation of protein content in eukaryotes via protein degradation, which involves three sequential reactions catalyzed by a ubiquitin-activating enzyme, a ubiquitin-conjugating enzyme (E2) and a ubiquitin-protein ligase (E3) (10). In eukaryotic cells, protein degradation is a highly selective and efficient ATP-dependent process, which occurs in the cytoplasm and nucleus (17). Substrate proteins are ubiquitinated, which specifically target the substrate protein for degradation by the $26 \mathrm{~S}$ proteasome (18). Protein degradation regulates several cellular processes including cell cycle progression. SCF (Skpl-Cullin-F-box) is a multisubunit E3 ubiquitin-protein ligase complex, consisting of F-box proteins, Skp1, Cullins, Rbxl, Nedd8 and Skp2 $(13,18)$. Although RNAi is a powerful gene-silencing process, there are relatively few studies that have assessed the application of RNAi technology towards Skp2 gene regulation in endometrial cancer. The aim of the current study was to investigate the expression of Skp2 in endometrial cancer and examine the effect of RNAi-induced Skp2 inhibition on the cell cycle, apoptosis and proliferation of the endometrium cancer cell line HEC-1-A.

Cyclins are a family of regulatory proteins that control cell cycle progression. Cyclins bind to and activate cyclin-dependent kinases $(\mathrm{Cdk})$, which control cell cycle processes via the phosphorylation of its target substrate, consequently driving 
the cell through G1 to S and G2 to M phase (19). If the G1-S or G2-M cell cycle checkpoints are blocked, cell proliferation may be inhibited, resulting in slow cell growth (19). In normal cell cycle progression, cyclin D1 forms a complex with Cdk4. Cyclin D1:Cdk4 phosphorylates and inactivates the retinoblastoma (RB) protein during early $\mathrm{G} 1$ phase to release E2F transcription factors, which initiate DNA synthesis, enabling cell cycle progression from the G1 to $\mathrm{S}$ phase during cell proliferation (20). Cyclin D1 overexpression occurs in several types of malignant tumor and dysregulated cyclin D1 expression can lead to the abnormal regulation of RB by Cdk4 (21). In addition, cyclin D1 can induce the expression of cyclin $\mathrm{E}$ and the activation of the Cyclin E-Cdk-2 complex in resting cells, causing the abnormal initiation of the cell cycle, which leads to the persistent proliferation of malignant cells (19). Several studies have demonstrated that cyclin D1 is closely associated with the occurrence, development, metastasis, prognosis and recurrence of several types of tumor (22-24).

p27 specifically binds to various cyclin-Cdk complexes and their monomers, blocking RB phosphorylation and inhibiting cell proliferation (25). In addition, p27 is also involved in cell-cell adhesion, promoting cell differentiation and inducing apoptosis $(26,27)$. Several studies have demonstrated that the low expression of p27 occurs in several types of malignant tumor and is closely associated with tumor invasion, metastasis, staging and prognosis (28-30). In tumors, p27 is associated with a reduction or loss of protein expression, while gene mutations are rare (31). The low expression of p27 also attenuates the inhibition of positive regulatory cell cycle proteins, resulting in excessive cell proliferation and tumor development (32).

It is generally considered that $\mathrm{Skp} 2$ expression is negatively associated with p27 expression (33,34); however, Kim et al (35) reported that in 332 untreated patients with cervical cancer, there was no correlation between Skp2 and p27. Additionally, Handra-Luca et al (36) and Fagan-Solis et al (25) reported that there was no correlation between Skp2 and p27 in salivary gland epidermoid carcinoma and breast cancer, respectively. Several studies have also demonstrated that low expression levels of p27 in malignant tumors are associated with high expression levels of cyclin D1 (37-39), although the correlation between p27 and cyclin D1 remains unclear. A previous study revealed that p27 and cyclin D1 are negatively correlated (37); however, Jonason et al (40) reported that cyclin D1 has a specific function in the post-transcriptional regulation of $\mathrm{p} 27$. There may therefore be a feedback pathway between p27 and cyclin D1, to maintain a dynamic balance between the positive and negative regulators of the cell cycle. However, this feedback pathway may not be a unidirectional regulatory pathway, but rather, an intricate regulatory system distributed among various members. An abnormality in one or more of these members may be involved in tumor development and progression, however this remains to be confirmed (41).

In the current study, the application of lentivirus-mediated RNAi technology was used to inhibit Skp2 gene expression. RT-qPCR results revealed that the inhibition of Skp2 expression did not affect the mRNA expression of p27 and cyclin D1. However, downregulation of Skp2 increased the expression levels of p27 and decreased the expression levels of Cychin D1. Flow cytometry demonstrated that RNAi-induced
Skp2 inhibition exerts an anti-proliferative effect by inducing cell cycle arrest in the G2/M phase, with no evidence of apoptosis. The inactive $35 \mathrm{kDa}$ subunit (but not the cleaved, activated $17 \mathrm{kDa}$ subunit) of caspase-3 was detected. However, there was no difference in the protein expression of caspase-3 following RNAi-induced Skp2 inhibition compared with the control group. These results indicate that the inhibition of Skp2 expression does not promote apoptosis of HEC-1-A cells. Therefore, RNAi-induced Skp2 inhibition does not affect the mRNA expression level of $\mathrm{p} 27$, but it does upregulate its protein expression by reducing the ubiquitin degradation of $\mathrm{P} 27$ protein.

Tsvetkov et al (42) revealed that SCF-Skp2 binds to phosphorylated Thr187 in p27 to specifically target p27 for degradation via a ubiquitin-dependent process during cell-cycle progression. In the current study, the inhibition of Skp2 expression did not increase the protein expression of cyclin D1, but instead downregulated cyclin D1 expression, which indicated that cyclin D1 may not be a substrate of the SCF complex, but a protein closely associated with Skp2. A study by Carrano et al (43) revealed that no direct biochemical data obtained using a reconstituted in vitro ubiquitination system, was available to verify whether cyclin D1, p21 and E2F-1 were true Skp2 substrates. However, It was likely, that Skp2 targets other substrates, including p27, for ubiquitination (43). Furthermore, the previous study revealed that two different Skp2 antisense oligonucleotides decrease Skp2 expression and concomitantly increase the levels of endogenous p27 (43). The study reveals that the phosphatase and tensin homolog/phosphoinositide 3-kinase signaling pathway and Cyclin D1 control a novel pathway that regulates the assembly of the SCF-Skp2 complex by modulating cullin neddylation and cullin associated and neddylation dissociated 1 binding at the G1/S cell cycle transition (37). Cyclin D1 regulates cyclin-dependent kinase inhibitor 1B (CDKN1B) abundance at a post-translational level, inhibiting the Skp2 promoter, Skp2 abundance and inducing CDKN1B phosphorylation at Ser10 (41). Therefore, it was hypothesized that cyclin D1 downregulation may be due to the reduced stability of other factors in the regulatory network affected by Skp2 expression. In the current study, inhibition of Skp2 led to the upregulation of p27 and G2/M arrest, which in turn inhibited the proliferation of HEC-1-A cells. These results correlated with other previous studies $(44,45)$. Nakayama et al (44) demonstrated that Skp2 regulates the stability of p27, which participates in the regulation of $\mathrm{G} 2 / \mathrm{M}$ phase. Pagano et al (45) also revealed that p27 inhibited Cdk1 activity at the G2/M phase, which is an important regulatory factor for G2-M checkpoints. In addition to p27, several cell cycle regulatory proteins also serve as substrates for SCF complexes, including cyclin A, cyclin B, p21 and p53, which are degraded via this pathway and are regulators of the G2-M checkpoint (45). Wu et al (46) identified small molecule inhibitors specific to SCF-Skp2 activity using in silico screens targeted at the binding interface for $\mathrm{p} 27$. These compounds selectively inhibited Skp2-mediated p27 degradation by reducing p27 binding through key compound-receptor contacts (46). In cancer cells, these compounds induce p27 accumulation in a Skp2-dependent manner and promote cell-type specific blocks in the $\mathrm{G} 1$ or G2/M phases (46). Furthermore, although the expression of p27 was upregulated in the current study, cell 
apoptosis was not affected. This may be due to the inhibition of Skp2 affecting the stability of unknown factors, offsetting the pro-apoptotic effect of p27. RNAi-induced Skp2 inhibition affects the stability of downstream factors, but may also affect the stability of upstream factors via feedback regulation, thereby causing changes in multiple factors along the entire cell regulatory network.

The results of the current study demonstrated that lentivirus-mediated RNAi technology can effectively inhibit the expression of Skp2, thereby upregulating the protein expression of p27 and inhibiting the proliferation of human endometrial cancer HEC-1-A cells. The current study verified the use of in vitro experiments to assess the mechanism underlying endometrial cell growth via Skp2 inhibition. Therefore, these methods may be used to further investigate the role of Skp2 in tumorigenesis in future studies. Furthermore, Skp2 is thought to be a potential target for gene therapy in patients with endometrial cancer (47). However, multi-level and multi-factor interactions involved in cell proliferation, apoptosis and cycle regulation need to be examined further and considered in an integrative manner. In addition, as there may be several factors underlying disease etiology, the effect of Skp2 on the expression levels of cyclin D1 and p27 as well as on cell proliferation, apoptosis and cycle regulation, and the association between Skp2 expression and IGFR signaling require further study.

\section{Acknowledgements}

Not applicable.

\section{Funding}

The present study was supported by grants from the National Science Foundation of Fujian (grant nos. 2016J01428 and 2016J01491) and the Joint Funds for the Innovation of Science \& Technology (grant no. 2017Y9062).

\section{Availability of data and materials}

The datasets used and/or analyzed during the current study are available from the corresponding author on reasonable request.

\section{Authors' contributions}

HL, XZ and PS designed the study. HL and XZ collected the data. HL, GR, XS and XC analyzed the data. HL and GR prepared the manuscript and PS revised the manuscript. All authors have read and approved the final manuscript.

\section{Ethics approval and consent to participate}

Not applicable.

\section{Patient consent for publication}

Not applicable.

\section{Competing interests}

The authors declare that they have no competing interests.

\section{References}

1. Vanderstichele A, Neven P and Vergote I: Combined modality adjuvant therapy for high-risk endometrial cancer. Lancet Oncol 17: 1029-1030, 2016.

2. Wirth T, Parker N and Ylä-Herttuala S: History of gene therapy. Gene 525: 162-169, 2013.

3. Ezoe S, Matsumura I, Nakata S, Gale K, Ishihara K, Minegishi N, Machii T, Kitamura T, Yamamoto M, Enver T and Kanakura Y: GATA-2/estrogen receptor chimera regulates cytokine-dependent growth of hematopoietic cells through accumulation of p21(WAF1) and p27(Kip1) proteins. Blood 100: 3512-3520, 2002.

4. Bochis OV, Irimie A, Pichler M and Berindan-Neagoe I: The role of Skp2 and its substrate CDKN1B (p27) in colorectal cancer. J Gastrointestin Liver Dis 24: 225-234, 2015.

5. Wang Z, Inuzuka H, Zhong J, Liu P, Sarkar FH, Sun Y and Wei W: Identification of acetylationdependent regulatory mechanisms that govern the oncogenic functions of Skp2. Oncotarget 3: 1294-1300, 2012

6. Xu SY, Wang F, Wei G, Wang B, Yang JY, Huang YZ, Zhang L, Zheng F, Guo LY, Wang JN and Tang JM: S-phase kinase-associated protein 2 knockdown blocks colorectal cancer growth via regulation of both p27 and p16 expression. Cancer Gene Ther 20: 690-694, 2013

7. Chung YK, Chi-Hung Or R, Lu CH, Ouyang WT, Yang SY and Chang CC: Sulforaphane down-regulates SKP2 to stabilize p27(KIP1) for inducing antiproliferation in human colon adenocarcinoma cells. J Biosci Bioeng 119: 35-42, 2015.

8. Masumoto K and Kitagawa M: E3 ubiquitin ligases as molecular targets in human oral cancers. Curr Cancer Drug Targets 16: 130-135, 2016.

9. Pratheeshkumar P, Siraj AK, Divya SP, Parvathareddy SK, Begum R, Melosantos R, Al-Sobhi SS, Al-Dawish M, Al-Dayel F and Al-Kuraya KS: Downregulation of SKP2 in papillary thyroid cancer acts synergistically with TRAIL on inducing apoptosis via ROS. J Clin Endocrinol Metab 103: 1530-1544, 2018.

10. Zhang W, Cao L, Sun Z, Xu J, Tang L, Chen W, Luo J, Yang F, Wang Y and Guan X: Skp2 is over-expressed in breast cancer and promotes breast cancer cell proliferation. Cell Cycle 15: 1344-1351, 2016.

11. Yamada S, Yanamoto S, Naruse T, Matsushita Y, Takahashi H, Umeda M, Nemoto TK and Kurita H: Skp2 Regulates the expression of MMP-2 and MMP-9, and enhances the invasion potential of oral squamous cell carcinoma. Pathol Oncol Res 22: 625-632, 2016.

12. Zhang H, Kobayashi R, Galaktionov K and Beach D: p19Skp1 and p45Skp2 are essential elements of the cyclin A-CDK2 S phase kinase. Cell 82: 915-925, 1995.

13. Wirbelauer C, Sutterlüty H, Blondel M, Gstaiger M, Peter M, Reymond F and Krek W: The F-box protein Skp2 is a ubiquitylation target of a Cul1-based core ubiquitin ligase complex: Evidence for a role of Cul1 in the suppression of Skp2 expression in quiescent fibroblasts. EMBO J 19: 5362-5375, 2000.

14. Tiscornia G, Singer O and Verma IM: Production and purification of lentiviral vectors. Nat Protoc 1: 241-245, 2006.

15. Livak KJ and Schmittgen TD: Analysis of relative gene expression data using real-time quantitative PCR and the 2(-Delta Delta C(T)) method. Methods 25: 402-408, 2001.

16. Wu H, Chen Y, Liang J, Shi B, Wu G, Zhang Y, Wang D, Li R, Yi X, Zhang $\mathrm{H}$, et al: Hypomethylation-linked activation of PAX2 mediates tamoxifen-stimulated endometrial carcinogenesis. Nature 438: 981-987, 2005.

17. Peth A, Uchiki T and Goldberg AL: ATP-dependent steps in the binding of ubiquitin conjugates to the $26 \mathrm{~S}$ proteasome that commit to degradation. Mol Cell 40: 671-681, 2010.

18. Bornstein G, Ganoth D and Hershko A: Regulation of neddylation and deneddylation of cullin1 in SCFSkp2 ubiquitin ligase by F-box protein and substrate. Proc Natl Acad Sci USA 103: 11515-11520, 2006.

19. Stacey DW: Cyclin D1 serves as a cell cycle regulatory switch in actively proliferating cells. Curr Opin Cell Biol 15: 158-163, 2003.

20. Xiao B, Spencer J, Clements A, Ali-Khan N, Mittnacht S, Broceño C, Burghammer M, Perrakis A, Marmorstein R and Gamblin SJ: Crystal structure of the retinoblastoma tumor suppressor protein bound to E2F and the molecular basis of its regulation. Proc Natl Acad Sci USA 100: 2363-2368, 2003.

21. Ru Y, Chen XJ, Zhao ZW, Zhang PF, Feng SH, Gao Q, Gao SG and Feng XS: Cyclin D1 and p57 as biomarkers in differentiation, metastasis and prognosis of gastric cardia adenocarcinoma. Oncotarget 8: 73860-73870, 2017. 
22. Georgiadou D, Sergentanis TN, Sakellariou S, Filippakis GM Zagouri F, Vlachodimitropoulos D, Psaltopoulou T, Lazaris AC, Patsouris E and Zografos GC: Cyclin D1, p16(INK) (4A) and p27(Kip1) in pancreatic adenocarcinoma: Assessing prognostic implications through quantitative image analysis. APMIS 122: 1230-1239, 2014.

23. Yoo J, Jung JH, Lee MA, Seo KJ, Shim BY, Kim SH, Cho DG, Ahn MI, Kim CH, Cho KD, et al: Immunohistochemical analysis of non-small cell lung cancer: Correlation witll clinical parameters and prognosis. J Korean Med Sci 22: 318-325, 2007.

24. Fu ZJ, Ma ZY, Wang QR, Lei DP, Wang R, Liu CX and Pan XL: Overexpression of CyclinD1 and underexpression of p16 correlate with lymph node metastases in laryngeal squamous cell carcinoma in Chinese patients. Clin Exp Metastasis 25: 887-892, 2008.

25. Fagan-Solis KD, Pentecost BT, Gozgit JM, Bentley BA, Marconi SM, Otis CN, Anderton DL, Schneider SS and Arcaro KF: SKP2 overexpression is associated with increased serine 10 phosphorylation of p27 (pSer10p27) in triple-negative breast cancer. J Cell Physiol 229: 1160-1169, 2014.

26. Wang H, Chen H, Zhou H, Yu W and Lu Z: Cyclin-dependent kinase inhibitor 3 promotes cancer cell proliferation and tumorigenesis in nasopharyngeal carcinoma by targeting p27. Oncol Res 25: 1431-1440, 2017.

27. Biçer A, Orlando S, Islam ABMMK, Gallastegui E, Besson A, Aligué R, Bachs $\mathrm{O}$ and Pujol MJ: ChIP-Seq analysis identifies p27(Kip1)-target genes involved in cell adhesion and cell signalling in mouse embryonic fibroblasts. PLoS One 12: e0187891, 2017.

28. Diersch S, Wenzel P, Szameitat M, Eser P, Paul MC, Seidler B, Eser S, Messer M, Reichert M, Pagel P, et al: Efemp1 and p27(Kip1) modulate responsiveness of pancreatic cancer cells towards a dual PI3K/mTOR inhibitor in preclinical models. Oncotarget 4: 277-288, 2013.

29. Santala S, Talvensaari-Mattila A, Soini Y, Kuvaja P and Santala M: Cyclins A, B, E and p27 in endometrial endometrioid adenocarcinoma. Anticancer Res 36: 6467-6473, 2016.

30. Zhu L, Chiao CY, Enzer KG, Stankiewicz AJ, Faller DV and Dai Y: SIRT1 inactivation evokes antitumor activities in NSCLC through the tumor suppressor p27. Mol Cancer Res 13: 41-49, 2015.

31. Lindberg D, Akerström G and Westin G: Mutational analysis of p27 (CDKN1B) and p18 (CDKN2C) in sporadic pancreatic endocrine tumors argues against tumor-suppressor function. Neoplasia 9: 533-535, 2007.

32. Zhu CQ, Shih W, Ling CH and Tsao MS: Immunohistochemical markers of prognosis in non-small cell lung cancer: A review and proposal for a multiphase approach to marker evaluation. J Clin Pathol 59: 790-800, 2006.

33. Jung D, Khurana A, Roy D, Kalogera E, Bakkum-Gamez J, Chien J and Shridhar V: Quinacrine upregulates p21/p27 independent of p53 through autophagy-mediated downregulation of p62-Skp2 axis in ovarian cancer. Sci Rep 8: 2487, 2018.

34. Wang ST, Ho HJ,Lin JT, Shieh JJ and Wu CY: Simvastatin-induced cell cycle arrest through inhibition of STAT3/SKP2 axis and activation of AMPK to promote p27 and p21 accumulation in hepatocellular carcinoma cells. Cell Death Dis 8: e2626, 2017.
35. Kim JY, Lim SJ, Kim HJ, Shin E, Park K and Lee CM: Clinical significance of p27 and Skp2 protein expression in uterine cervical neoplasm. Int J Gynecol Pathol 26: 242-247, 2007.

36. Handra-Luca A, Ruhin B, Lesty C and Fouret P: P27, SKP2, and extra-cellular signal-related kinase signalling in human salivary gland mucoepidermoid carcinoma. Oral Oncol 42: 1005-1010, 2006.

37. Guan G, Bakr MM, Firth N and Love RM: Expression of cyclin D1 correlates with $\mathrm{p} 27^{\mathrm{KIP} 1}$ and regulates the degree of oral dysplasia and squamous cell carcinoma differentiation. Oral Surg Oral Med Oral Pathol Oral Radiol 126: 174-183, 2018.

38. Lee KH, Lee HE, Cho SJ, Cho YJ, Lee HS, Kim JH, Nam SY, Chang MS, Kim WH and Lee BL: Immunohistochemical analysis of cell cycle-related molecules in gastric carcinoma: Prognostic significance, correlation with clinicopathological parameters, proliferation and apoptosis. Pathobiology 75: 364-372, 2008.

39. Pesutić-Pisac V, Punda A, Gluncić I, Bedeković V, Pranić-Kragić A and Kunac N: Cyclin D1 and p27 expression as prognostic factor in papillary carcinoma of thyroid: Association with clinicopathological parameters. Croat Med J 49: 643-649, 2008.

40. Jonason JH, Gavrilova N, Wu M, Zhang H and Sun H: Regulation of SCF(SKP2) ubiquitin E3 ligase assembly and p27(KIP1) proteolysis by the PTEN pathway and cyclin D1. Cell Cycle 6: 951-961, 2007.

41. Li Z, Jiao X, Wang C, Ju X, Lu Y, Yuan L, Lisanti MP, Katiyar S and Pestell RG: Cyclin D1 induction of cellular migration requires p27(KIP1). Cancer Res 66: 9986-9994, 2006.

42. Tsvetkov LM, Yeh KH, Lee SJ, Sun H and Zhang H: p27(Kip1) ubiquitination and degradation is regulated by the $\mathrm{SCF}(\mathrm{Skp} 2)$ complex through phosphorylated Thr187 in p27. Curr Biol 9: 661-664, 1999

43. Carrano AC, Eytan E, Hershko A and Pagano M: SKP2 is required for ubiquitin-mediated degradation of the $\mathrm{CDK}$ inhibitor p27. Nat Cell Biol 1: 193-199, 1999.

44. Nakayama K, Nagahama H, Minamishima YA, Miyake S, Ishida N, Hatakeyama S, Kitagawa M, Iemura S, Natsume T and Nakayama KI: Skp2-mediated degradation of p27 regulates progression into mitosis. Dev Cell 6: 661-672, 2004.

45. Pagano M: Control of DNA synthesis and mitosis by the Skp2-p27-Cdk1/2 axis. Mol Cell 14: 414-416, 2004.

46. Wu L, Grigoryan AV, Li Y, Hao B, Pagano M and Cardozo TJ: Specific small molecule inhibitors of Skp2-mediated p27 degradation. Chem Biol 19: 1515-1524, 2012.

47. Pavlides SC, Huang KT, Reid DA, Wu L, Blank SV, Mittal K, Guo L, Rothenberg E, Rueda B, Cardozo T and Gold LI: Inhibitors of SCF-Skp2/Cks1 E3 ligase block estrogeninduced growth stimulation and degradation of nuclear p27kip1: Therapeutic potential for endometrial cancer. Endocrinology 154: 4030-4545, 2013. 